\title{
Some Wave Simulation Properties of the $(2+1)$ Dimensional Breaking Soliton Equation
}

\author{
Emine Nesligül Aksan ${ }^{1, *}$, Hasan Bulut ${ }^{2}$, and Miraç Kayhan ${ }^{1}$ \\ ${ }^{1}$ Department of Mathematics, University of İnonu, Malatya, Turkey \\ ${ }^{2}$ Department of Mathematics, University of Firat, Elazı ̆̆, Turkey
}

\begin{abstract}
In this paper, we apply an efficient method which is improved Bernoulli sub-equation function method (IBSEFM) to $(2+1)$ dimensional Breaking Soliton equation. It gives some new wave simulations like complex and exponential structures. We test whether all structures verify the $(2+1)$ dimensional Breaking Soliton model. Then, we draw three and two dimensional plane by using Wolfram Mathematica 9.
\end{abstract}

\section{Introduction}

Every branch of non-linear partial differential equations (NLPDEs) in applied science plays an major role such as chemical physics, chemical kinematics, optical fibers, fluid mechanics so on. The only wave solutions to the non-linear partial differential equation have been used to describe some physical phenomena. In the literature, plenty of effective methods have been improved to discover the only wave solutions of NLPDEs. Some of those methods are Sine-Gordon expansion method, Homotopy analysis method, The modified simple equation method, generalized Bernoulli sub-equation method, improved Bernoulli sub-equation method. In this article we handle $(2+1)$ dimensional Breaking Soliton Equation [2] defined as:

$$
u_{x t}-u_{x x x y}-2 u_{x x} u_{y}-4 u_{x} u_{x y}=0
$$

Xie gained non-travelling wave solutions by the developed tanh function method, that they introduced a generalized Riccati equation and obtained different solutions. In this article, we built new non-travelling wave solutions of partial differential equations. Last of all, new non-travelling wave solutions including solution and cyclical solution of PDE are gained. A generalized flexible-coefficent algebraic method with computerized emblamatic calculation is improved to handle $(2+1)$ dimensional Breaking Soliton equation with flexible parameter. Chen al. work the $(2+1)$ Breaking Soliton equation by using the new generalized transformation in homogeneous balance method.

\footnotetext{
* Corresponding author: mirackayhan@yandex.com
} 


\section{Fundamental Properties of the Method}

IBSEFM created with extending BSEFM [5,6] will be offered in this sub-division. Hence, we take account of the follows.

Step 1. We think following PDE;

$$
P\left(u, u_{x}, u_{y}, u_{z}, u_{t}, u_{t t}, u_{x t}, u_{x x}, u_{x y}, u_{y y}, u_{y t}, u_{z z}, \ldots\right)=0,
$$

and get the wave conversion

$$
u(x, y, z, t)=U(\eta), \eta=k_{1} x+k_{2} y+\lambda t .
$$

If equation (3) is written in equation (2) transform a (NODE) as following;

$$
N\left(U, U^{\prime}, U^{\prime \prime}, U^{\prime \prime \prime}, \cdots\right)=0 \text {. }
$$

Step 2. Thinking test equation of results in equation (2), it can be written as following;

$$
U(\eta)=\frac{\sum_{i=0}^{n} a_{i} F^{i}}{\sum_{j=0}^{m} b_{j} F^{j}}=\frac{a_{0}+a_{1} F+a_{2} F^{2}+\cdots+a_{n} F^{n}}{b_{0}+b_{1} F+b_{2} F^{2}+\cdots+b_{m} F^{m}},
$$

and with respect to the Bernoulli theorem,

$$
F^{\prime}=\sigma F+d F^{M}, w \neq 0, d \neq 0, M \in \mathbb{R}-\{0,1,2\},
$$

where $F=F(\eta)$ is Bernoulli differential polynom. Take place (6) connection in equation(4), it provide us an equation of polynom $\Omega(F)$ of $F$;

$$
\Omega(F)=\rho_{s} F^{s}+\cdots+\rho_{1} F+\rho_{0}=0 .
$$

With respect to the balance essence, we able to gain the values of $n, m$ and $M$.

Step 3. Let parameter of $\Omega(F)$ must have zero will provide equations system;

$$
\rho_{i}=0, i=0, \cdots, s .
$$

Solve the system, we going to define the values of $a_{0}, \cdots, a_{n}$ and $b_{0}, \cdots, b_{m}$.

Step 4. When we solving non-linear Bernoulli differential equation Eq.(6), we gain two cases with respect to $\sigma$ and $d$ conditions as following;

$$
\begin{gathered}
F(\eta)=\left[\frac{-d}{\sigma}+\frac{\tau}{e^{\sigma(M-1) \eta}}\right]^{\frac{1}{1-M}}, \sigma \neq d, \\
F(\eta)=\left[\frac{(\tau-1)+(\tau+1) \tanh \left(\frac{\sigma(1-M) \eta}{2}\right)}{1-\tanh \left(\frac{\sigma(1-M) \eta}{2}\right)}\right]^{\frac{1}{1-M}}, \sigma=d, \quad \tau \in \mathbb{R} .
\end{gathered}
$$

Using a whole distinction system for polynom of $F$, we analyse equation (8) by using of Mathematica 9 and partition the exact solutions to equation (4). To interpretations better results gained in this wise, we are able to draw $3 \mathrm{D}$ and $2 \mathrm{D}$ shape of the results gained by taking suitable coefficients. 


\section{Application}

In this subdivision of manuscript, we supply some experimental solutions to describe the demonstration of the analytic algorithm suggested.

If transformation from Eq.(3) to Eq.(1), is applied;

$$
\lambda U^{\prime \prime}-k_{1}^{2} k_{2} U^{(4)}-6 k_{1} k_{2} U^{\prime \prime} U^{\prime}
$$

Integrating and arrangement the values of integration to zero and $V=U^{\prime}$ written, we gain

$$
\lambda V-k_{1}^{2} k_{2} V^{\prime \prime}-3 k_{1} k_{2} V^{2}=0
$$

Balancing $U^{\prime \prime}$ with $U^{2}$ in (12) gives

$$
2 M+m=n+2 \text {. }
$$

If we take $M=3, m=1, n=5$ for Eq. (13), after that, we can write the following equations;

$$
\begin{gathered}
U(\eta)=\frac{a_{0}+a_{1} F(\eta)+a_{2} F^{2}(\eta)+a_{3} F^{3}(\eta)+a_{4} F^{4}(\eta)+a_{5} F^{5}(\eta)}{b_{0}+b_{1} F(\eta)}=\frac{\Upsilon(\eta)}{\Psi(\eta)} \\
U^{\prime}(\eta)=\frac{\Upsilon^{\prime}(\eta) \Psi(\eta)-\Upsilon(\eta) \Psi^{\prime}(\eta)}{\Psi^{2}(\eta)}
\end{gathered}
$$

and

$U^{\prime \prime}(\eta)=\frac{\Upsilon^{\prime \prime}(\eta) \Psi(\eta)-\Upsilon(\eta) \Psi^{\prime}(\eta)}{\Psi^{2}(\eta)}-\frac{\left[\Upsilon(\eta) \Psi^{\prime}(\eta)\right]^{\prime} \Psi^{2}(\eta)-2 \Upsilon(\eta)\left[\Psi^{\prime}(\eta)\right]^{2} \Psi(\eta)}{\Psi^{4}(\eta)}$

where $F^{\prime}=w F+d F^{3}, w \neq 0, d \neq 0$. When we substitutes Eqs.(14,16) in the Eq.(12), we obtain a system of equations for Eq.(12). Hence, we achieve an equation from the parameters of polynom of $F$. By solving equation by using of Wolfram Mathematica 9, it yields the following coefficients;

Case 1. For $\sigma \neq d$, we can find following parameters:

When we substitute these parameters with Eq.(3) in Eq.(14), we gain some new exponential, complex and hyperbolic function solutions to the Eq.(1) as following, respectively;

Case 1. 1. For $b \neq d$, it imaginable following parameters;

$a_{2}=\frac{4 d \sqrt{\lambda} b_{0}}{\sqrt{k_{1}}}, a_{3}=\frac{4 d \sqrt{\lambda} b_{1}}{\sqrt{k_{1}}}, a_{4}=-8 d^{2} b_{0} k_{1}, a_{5}=8 d^{2} b_{1} k_{1}, \sigma=\frac{-\sqrt{\lambda}}{2 k_{1} \sqrt{k_{2}}}$

Substituting these parameters in Eq.(14) with Eq.(9), we find the following exponential 
function solution for Eq.(1);

$$
u_{1}(x, y, t)=-\frac{4 d \sqrt{\lambda} k_{1}}{e^{f(x, y, t)} \tau \sqrt{\lambda}+2 d k_{1} \sqrt{k_{2}}} .
$$

Where $f(x, y, t)=\frac{\sqrt{\lambda}\left(t \lambda+x k_{1}+y k_{2}\right)}{k_{1} \sqrt{k_{2}}}$.

When we consider the suitable values of parameters, we can find new wave simulations for Eq.(1) as following figures:
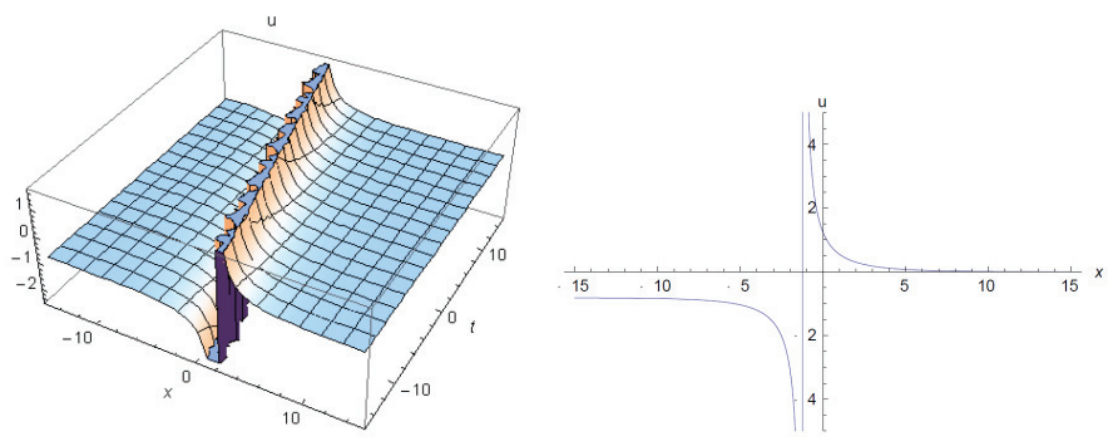

Fig.1. The three dimensional and two dimensional planes of the travelling wave results Eq.(18) by considering the values

$\lambda=0.1, d=0.3, y=0.4, k_{1}=0.5, k_{2}=0.6, \tau=-1 \quad-15<x<15,-5<t<5$, and $t=0.02$

Case 1. 2. For $b \neq d$, it imaginable following parameters;

$$
\begin{aligned}
& a_{0}=\frac{\lambda b_{0}}{3 k_{1} k_{2}}, a_{1}=\frac{\lambda b_{1}}{3 k_{1} k_{2}}, a_{2}=\frac{4 i d \sqrt{\lambda} b_{0}}{\sqrt{k_{2}}}, a_{3}=\frac{4 i d \sqrt{\lambda} b_{1}}{\sqrt{k_{2}}}, a_{4}=-8 d^{2} b_{0} k_{1} \\
& a_{5}=-8 d^{2} b_{1} k_{1}, \sigma=-\frac{i \sqrt{\lambda}}{2 k_{1} k_{2}} .
\end{aligned}
$$

Substituting these parameters in Eq.(14) with Eq.(9), we reach the following exponential function solution for Eq.(1);

$$
u_{2}=\frac{4 d \sqrt{\lambda} k_{1}}{-e^{\frac{i \sqrt{\lambda} f(x, y, t)}{k_{1} \sqrt{k_{2}}}} \tau \sqrt{\lambda}+2 i d k_{1} \sqrt{k_{2}}}+\frac{\lambda f(x, y, t)}{3 k_{1} k_{2}},
$$

where a $f(x, y, t)=t \lambda+x k_{1}+y k_{2}$. When we consider the suitable values of parameters, we can find new wave simulations for Eq.(1) as following figures: 

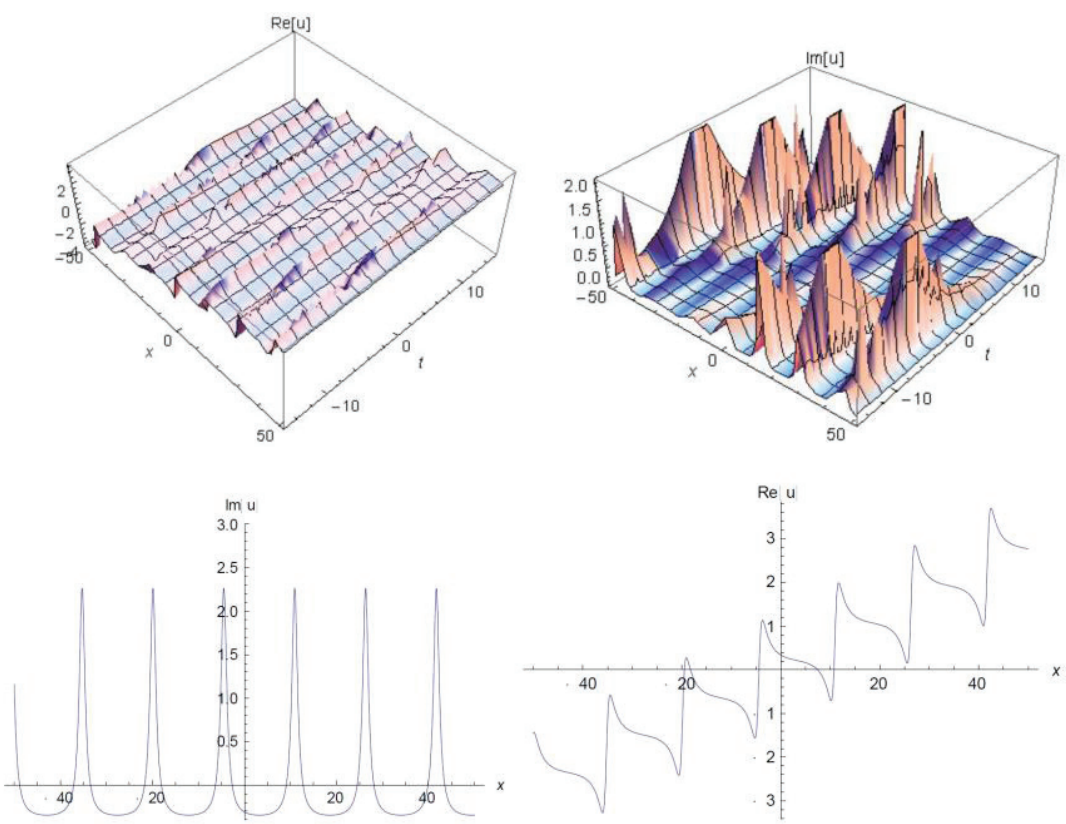

Fig.2. The three dimensional and two dimensional planes of the travelling wave results Eq.(20) by considering the values

$$
\lambda=0.1, d=0.3, y=0.4, k_{1}=0.5, k_{2}=0.6, \tau=-1-50<x<50,-0.4<t<3 \text { and } t=0.7
$$

\section{Conclusion}

In this paper, IBSEFM has been applied to $(2+1)$ dimensional Breaking Soliton Equation. We have gained some new traveling wave results like complex and exponential function solutions. According to new results and all figures, it has been observed that this way method is a efficient tool for solving such mathematical models.

\section{References}

1. M. T. Darvishi, M. Najafi, World Ac. of Sci. 55, 919-922 (2011)

2. M. T. Darvishi, M. Najafi, Int. J. of Appl. Mat, 1, 1-7 (2012)

3. Giese, R. Bjerkving, M. Westphal, J. Clin. Oncol, 21(8), 1624(2003)

4. H. Bhrawy, M. A. Abdelkawy, A. Biswas. Indian J. Phys, 87,1125-1131 (2013)

5. H.M. Baskonus, H. Bulut, Waves in Rnd. and Comp. Med, 26, 189-196 (2016)

6. H.M. Baskonus, 1st Int. Sym. on Co.Mat. Sci. Morocco(2016)

7. F. Özpinar, H. M. Baskonus and H. Bulut, Entropy, 17(12), 8267-8277 (2015)

8. H.M. Baskonus, H. Bulut, Waves in Rnd. and Comp. Med, 25(4), 720-728 (2015)

9. Bekir, F. Uygun, Arab J. of Mat. Sci. 18, 73-85 (2012)

10. A.J.M. Jawad, M.D. Petkovic and A. Biswas, Appl. Math. Comput.,217, 869-877 (2010)

11. F.B.M., Belgacem, 2010. Appl. Mat. Sci. 4 (74), 3665-3686.

12. F. Belgacem, A.A. Karaballi, and S.L. Kalla, Math. Prob. Eng., 3-4,103 (2003) 